Lithotomy with a rectangular staff; p. 406.

Two cases of fistulous opening in the urethra in front of the scrotum : one of which was cured by repeated plastic operations ; the other partially cured; p. 981 .

VIII. Injuries and Diseases of the Organs of Generation, and of the Female Breast.

In this class will be found-

A case of displacement, or dislocation, of the testicle from injury; p. 549

Ifydrocele containing spermatozoa, and having an unusual re. lation to the testicle; p. 81 .

Strumous disease of the testicle; p. 223.

$A$ case of fibrous tumour of the uterus extirpated by gastrotorny; p. 159

Operation for prolapsus uteri, followed by symptoms of pyæmia; p. 643 .

A case of ovarian disease in which tapping was followed by effusion of blood into the cyst and peritonitis; p. 140 .

A case of ovarian dropsy in which the cyst was found closely adherent in the pelvis to the rectum and vagina; and had pressed on the ureters, causing absorption of the kidneys; p. $2(60$.

$\Lambda$ case of ovarian dropsy where the cyst communicated freely with the peritoneal carity; p. 941 .

Cases of successful ovariotomy; pp. $76 \pm, 1001$.

Numerous cases of vesico-vaginal fistula, most of them operated on with success; pp. 901, $921,942,1041$

Vaginal cystocele; p. 575.

Case of epithelial cancer of the male generative organs and urethra, with stricture: perinæal section : p. 301 .

Warts of the labia; p. 492 .

IX. Affections of the Blood-Vessels.

This head includes-

$A$ series of numerous cases of aneurism; pp. 42, 61, 82, 142, 200,260

Two cases of aneurism of the aorta; p. 240.

A case of aneurism of the aorta, which burst into the pericardium; p. 100.

A case of ruptured popliteal aneurism, for which amputation was necessary; another aneurism formed in the femoral artery. The result was fatal; pp. 302, 447, 530 .

A case of aneurism of the external iliac artery, presenting unusual difficulties of diagnosis; p. 724

A case of vascular tumour; p. 201 .

$\Lambda$ neurism by anastomosis on the scalp, treated by the galvanic cautery and other local measures, apparently with success ; p. 491.

A case of varicose veins, treated with potassa fusa, followed by pyæmia; p. 921 .

X. Injuries and Diseases of the Bones and Jornts. In this section will be found-

A case of compound comminuted fracture of the femur; p. 41 . Compound fracture of the leg; p. 222.

Forcible straightening of a badly set fracture; p. 902.

Dislocation and fracture of the carpus; p. 882 .

Case of forcible extension of ankylosis; p. 62.

Congenital dislocation of the bip; p. 962.

Amputation on account of abscess in the head of the tibia; $p$. 161 .

Amputation for ulceration of the cartilages; p. 222.

Case illustrating the possibility of mobility at the knee after excision with a useful limb; p. 1001.

Case of housemaid's knee; p. 6 ; 7 .

Hydrarthrosis of the knee.joint injected with iodine; p. 119.

Latent psoas abscess; p. 529.

\section{Constitutional Diseases.}

The cases included in this section, besides those which will be found under the previous heads, are-

(a) Cancer:-

Cases illustrating the treatment of cancer with caustics; pp. 142,180 .

Cancer of the sole of the foot; p. 141.

Epithelial cancer of the face; p. 181.

Two cases of malignant tumour of the leg; p. 061.

(b) Syphilis:-

A series of cases, in which syphilis was treated by calomel fumigation, is found at pp. 281, 327, 595, 678 .

(c) Pyamia. The rare phenomenon of the occurrence of pyæmia in a child, and secondary deposit in the walls of the heart, is illustrated at p. 406 . (d) Scrofula. Extensive scrofulous deposits in various parts of the body are noticed at pp. 240,347 ; and a case of abscess and unhealthy sores in various parts, which proceeded from some condition of the blood allied to scrofula, at p. 1041.

(e) Tetanus. The cases at pp. 328, 385, 406, 550, 882, illustrate various points in the pathology and treatment of this disease.

\section{Operative Surgery.}

This class consists of cases reported principally with a view to the operation, and is exclusive of numerous other cases in which operations were performed, but which are to be found in other classes.

Amputation at the knee-joint; p. 961.

Teale's amputation; p. 962.

Excision of the shoulder; p. 42.

Partial excision of the upper jaw ; p. 101

Excision of the upper jaw for fibrous tumours of the pharynx pp. $61,83,446$.

Excision of the elbow; p. 179.

Excision of the head of the femur and tuber ischii; p. 239 .

Excision of the knee; p. 285.

State of the knee-joint after excision; p. 1001.

Excision of the hip; p. 365.

Partial amputation of the foot; p. 472 .

Iisfranc's amputation; p. 725.

Syme's amputation; p. 784 .

Removal of a tumour below the jaw; p. 63 .

Operation on the left colon (gastrotomy); p. 1021

Ovariotomy ; p. 1001.

A case showing the permanence of the operative cure of prolapsus uteri ; p. 815.

XiII. New Inventions: Miscellaneous.

Under this head will be found the following cases:-

Use of plaster of Paris bandage; p. 161.

Use of ether as an anæsthetic; p. 575

New splint for fractured jaw ; p. 845.

Use of silver sutures; p. 942.

Chloroform as a narcotic; p. 328.

Cases of poisoning with stramonium ; p. 823.

Cystic tumours of the neck; p. 863.

Hydrocele of the neck; p. 446.

Suppurating cyst of the neck; p. 222 .

Sebaceous tumour absorbing the skull ; p. 783 .

Removal of glandular tumours pressing on the trachea; p. 677 . Cases of ganglion beneath the radial artery; p. 142.

Case of fibro-plastic tumour originating in a ganglion; $p$. 239,901 .

Case of muscular atrophy; p. 346

Treatment of hysterical contraction of the fingers by "firing" p. 63.

Case of wound of the abdomen with a piece of wood; p. 120

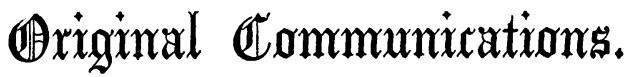

\section{ON NAVUS.}

By J. C. Wordsworth, Esq., Assistant-Surgeon to the London Hospital.

To the conscientious practitioner of our art, it is at all times a matter of anxious consideration to decide how much he may entrust to nature to accomplish in her own way the cure of disease, and when it becomes his duty to interrupt or supersede her processes, by the intervention of his own expedients.

As science progresses, I believe it may be said that we become less obtrusive as practitioners, and learn to confide more in nature's resources. Hence we see that surgeons, actuated by the accumulated experience of ages, are daily more disposed to limit their interference to the obvious necessities of treatment. Whoéver reflects on the tendency of modern medicine, must be impressed by the growing indisposition to in. trude the imperfect dictates of our art needlessly and rashly, when, on the one hand, nature does not need our aid, or on the other, she does not vouchsafe ns her confidence.

It would prove a most interesting and instructive indication of the progress of science, to review and contrast the ideas of different ages of the world, on the treatment of disease. I believe that the comparison would show a gradually progressive 
disposition to abandon officious interference, pari passu with the advance of our knowledge of disease. This tendency to follow only the plain indications of nature, has culminated in our times, and originated the so-called " school of expectancy." Much good will doubtless flow from this tide in medical science, and its recession will leave us in possession of still greater confidence in the powers of nature to repair the lesions of our organisms. There is no fear of our degenerating into homocopathy, but, at the same time, we shall learn still more to direct our efforts into the course which nature indicates, so as to aid and not oppose her labours.

We shall also be disposed attentively to watch the tendencies of disease, and so draw our conclusions as to the necessity of our cooperation, as well as to see when we can most opportunely act. It will also follow as a corollary, that we shall still more restrict operative proceedings to such cases of disease, as are either quite out of the reach of natural remedy, or are but imperfectly or doubtfully repaired by the unaided efforts of nature.

Conservative surgery is daily teaching us to limit our proceedings to the minimum required, and that we had better err on the side of deficiency than of excess. If it be demanded of us to demonstrate such progress in the art of healing as has distinguished other branches of human knowledge, we can triumphantly adduce examples of our improved practice in the treatment of diseased joints, of congenital abnormities, and of lesions which only modern surgery has ventured to improve. Much has been done, and is in progress, yet doubtless more remains to stimulate our efforts. Guided by the scientific spirit of our times, there is much prospect of an abundant harvest from the labours of those who are cultivating medicine as a branch of inductive philosophy, for though we are really not in possession of the certain data which form the basis of more exact sciences, we have abundance to preserve us from scepticism.

I have made these few remarks as a preface to the consideration of a subject to which I believe they are especially pertinen-I mean the modern treatment of that class of diseases cornprised in the expression " vascular tumours."

It seldom happens that a month passes without some instances of nævus occurring in the practice of the hospitals to which I am attached, and I generally find that the friends of the patients are fully prepared for operations, as a matter of course. When a nævus is placed on one of its favourite localities, such as the scalp or face, the common impression appears to be that it must necessarily spread, and that therefore the sooner it is submitted to operation the better.

But let us see what is likely to become of the tumour if it escape the zeal of the surgeon.

It will be found that there is not always so much need of precipitancy as some would have us suppose. For some time, perhaps a few weeks, the navus will continue to grow, rather rapidly for a time, afterwards more slowly; till it acquires the magnitude of a penny piece. It then ceases to grow, usually ; but sometimes acquires a much larger size. It has usually a well-defined limit, which is seldom much exceeded. If of the simple, cutaneous variety, it may become the seat of eczema, or of superficial ulceration, spreading in all directions. This may be its condition for some months, and it then gradually ceases and cicatrisation occurs. Scarcely a trace of tumour may be seen; over its site, if on the scalp, hair grows, of a darker colour, coarser texture, and more thickly set than elsewhere. This is a common course for the tumour to take. In a second series of cases, I have found the cutaneous nævus grow to a certain limit, then dwindle and degenerate, so as to leave 8 pale and scarred surface with little or no remnants of its vas. cular elements apparent. In a third class, the nævus grows to the limit above-named, and then ceases to increase; the vessels remain permanently, and it forms a more or less elevated tumour, of a dark red or purple colour, on which hair may grow, but usually only scantily.

In the subcutaneous or areolar variety, the above results are less common, and unless the skin be involved, I have never seen them spontaneously disappear. They usually remain as soft spongy masses for some years, and may perhaps degenerate and be absorbed during youth. Of this, I am only able to speak with probability, as I have not yet had actual proof but judging from their rarity in mid-life, I am led to this conclusion; for surely all are not operated on. The spontaneous cure, then, is not attended by any danger, nor by much inconvenience. Some irritation accompanies the eczema or ulcera tion, and occasionally a little blood is lost. From the abovestated considerations, I conclude that it is not necessary to in. terfere with the common kinds of nævus, unless they are so placed as to produce great deformity, or they are likely to involve important parts, such, for instance, as the eyelids. When they commence deeply, there appears no limit to their growth. They then become one of the most formidable affairs with which the surgeon has to deal. I have lately seen one in the orbit, which has been treated by Dr. Mott of New York; in this case, the carotid artery has been ligatured, without producing a cure. He is now under the care of my colleague, Mr. Poland, in the Ophthalmic Hospital. One occurred in the practice of my colleague, Mr. Adams, at the London Hospital, some time aro, in which the whole lower limb was implicated. Mr. Critchett, some years ago, tied the carotid artery for one in the orbit, at the London Hospital. The disease was checked for a time, but returned.

I remember instances in which fatal results have followed operations on small superficial nevi in children, when in all probability the diseases would have disappeared had they been entrusted to nature's remedy. But when successfully treated by ligature or other means, much agony is necessarily inflicted in the process, and $I$ have known in my own practice many a little creature reduced from a state of ruddy health to one of great danger, through the operation of the ligature. For these reasons, I never operate on them till I have had some opportunity of observing their progress. Since I came to the decision that the operation is seldom required, I have had very few occasions to depart from the practice, though I have frequently six or eight cases under observation at one time.

So much have I found the friends of children actuated by a desire to submit them to operation, that the principal difficulty I have had to contend with, has been their reluctance to give a fair trial to the course which the tumours naturally take.

I have lately had under observation a case of cutaneous nævus of the scalp, as large as a crown piece, and situate on the occiput. The subject being a baby of a few months. In this instance, a few small vessels were noticed soon after the child's birth, apparently involving the thickness of the scalp. After a few weelss it commenced to spread circularly, and in six months had acquired its greatest extent. It then became affected by an eruption of eczematous character, and small points of ulceration from time to time crept along its surface, producing some irritation, and disturbing the child's rest at night. A lotion of the dilute solution of diacetate of lead reduced the irritation, and under its influence the vessels gradually disappeared, the surface completely healed, and there is not a vestige of it apparent at this time. A lock of dark, rather coarse and curly hair now marks the seat of the growth, the child being eighteen months old.

Several months ago I was requested by my friend Dr. Hess to see a nævus on the body of one of his children. It had acquired the usual size, and was of the subcutaneous variety After many months he tells me it has not increased, but remains a soft spongy mass, having apparently little disposition to change. No treatment has been adopted.

A case has been recently under my care at the London Hospital. It was a subcutaneous nævus on the forehead of a child, of about the size of a half-crown, and rising considerably fiom the surface, produced an amount of deformity which called for some interference. I thercfore passed two ligatures subcutaneously through and around the tumour, encrrcling it in two halves. In a few days both ligatures came away, and the tumour gradually shrank, leaving no leformity.

Another case has occurred amongst my out-patients at the Royal London Ophthalmic Hospital, which, from its situation and important relations, required the interference of art.

It occupied the upper eyelid of a child, implicating the entire breadth of the lid, and consequently producing an amount of deformity which was dangerous to the eye. Chloroform was administered to the child, and the lid was punctured in many places by a hot needle, so as to set up considerable inflammation and exudation. The nævus was completely destroyed, leaving scarcely a vestige of its peculiar structure, and at the same time restoring the motions of the lid to their normal condition.

The above examples (from a considerable number of which I have notes) are sufficient to illustrate my view that a very large proportion of nævi are unnecessarily submitted to operation, when placed on parts of the body where their presence is neither prejudicial to function nor productive of serious deformity.

41, Finsbury Square, November, 1858.

(1, 